\title{
THE MINIMUM MODULUS OF CERTAIN SMALL ENTIRE FUNCTIONS
}

BY

P. C. FENTON

ABSTRACT. Suppose that $f(z)$ is an entire function satisfying

$$
\min _{|z|=r}|f(z)| \leqslant C(\sigma) \max _{|z|=r}|f(z)|,
$$

for $r \geqslant \rho_{0}>0$, where $\sigma>0$ and

$$
C(\sigma)=\prod_{k=1}^{\infty}\left\{\frac{1-\exp (-(2 k-1) / 4 \sigma)}{1+\exp (-(2 k-1) / 4 \sigma)}\right\}^{2} .
$$

It is shown that

$$
\lim _{r \rightarrow \infty} \frac{\max _{|z|=r}|f(z)|-\sigma(\log r)^{2}}{\log r} \geqslant-2 \sigma \log \left(\max \left(\rho_{0},\left|a_{1}\right|\right)\right),
$$

where $a_{1}$ is the first nonzero zero of $f$.

1. Introduction and result. Suppose that $f(z)$ is an entire function and that $M(r, f)=\max _{|z|=r}|f(z)|, m(r, f)=\min _{|z|=r}|f(z)|$. In [1] P. D. Barry conjectured that if

$$
\varlimsup_{r \rightarrow \infty} \frac{\log M(r, f)}{(\log r)^{2}}=\sigma<+\infty
$$

then, for a sequence of $r \rightarrow \infty$,

$$
\frac{m(r, f)}{M(r, f)}>\prod_{k=1}^{\infty}\left\{\frac{1-\exp (-(2 k-1) / 4 \sigma)}{1+\exp (-(2 k-1) / 4 \sigma)}\right\}^{2}+o(1) .
$$

This was proved by the author in [3] and, under more general conditions, by A. A. Gol'dberg [4]. In fact Gol'dberg proved that if $f$ has zero order and if

$$
\lim _{r \rightarrow \infty} \frac{1}{(\log r)^{2}} \int_{0}^{r} n(t, f) \frac{d t}{t} \leqslant \sigma<+\infty,
$$

then (1.2) holds on a sequence of $r \rightarrow \infty$. Here $n(t, f)$ is the number of zeros of $f$ in $|z|<t$, assuming (as may be done without loss of generality) that $f(0) \neq 0$. It happens that by using estimates developed by Kjellberg [6], the proof in [3] may be

Received by the editors December 9, 1980.

1980 Mathematics Subject Classification. Primary 30A64. 
altered in a straightforward way to show that (1.2) holds on a sequence of $r \rightarrow \infty$ if only

$$
\lim _{r \rightarrow \infty} \frac{\log M(r, f)}{(\log r)^{2}}=\sigma<+\infty
$$

We shall prove here a sharpened version of this result.

THEOREM. Suppose that $\sigma$ is any positive number and that $f(z)$ is an entire function such that

$$
\frac{m(r, f)}{M(r, f)} \leqslant C(\sigma)=\prod_{k=1}^{\infty}\left\{\frac{1-\exp (-(2 k-1) / 4 \sigma)}{1+\exp (-(2 k-1) / 4 \sigma)}\right\}^{2}
$$

for all $r \geqslant \rho_{0}>0$. Then

$$
\lim _{r \rightarrow \infty} \frac{\log M(r, f)-\sigma(\log r)^{2}}{\log r} \geqslant-2 \sigma \log \left(\max \left(\rho_{0},\left|a_{1}\right|\right)\right),
$$

where $a_{1}$ is the first nonzero zero of $f$.

An earlier result of the author [2] suggests that if the lower limit in (1.4) is finite then

$$
\lim _{r \rightarrow \infty} \frac{\log M(r, f)-\sigma(\log r)^{2}}{\log r}
$$

ought to exist. While this remains a firm possibility nevertheless the proof of the theorem here proceeds by smoothing out the distribution of the zeros of $f$, and this inevitably involves a loss corresponding (more or less) to the difference between the left- and right-hand sides of (1.4). To be a little more specific, if $f(z)$ is a function with positive zeros satisfying (1.3) and (1.4), with the left-hand side of (1.4) finite, then any function obtained from $f$ by adding to it a finite number of positive zeros will also satisfy (1.3) and (1.4); and our proof is unable to distinguish between the two functions. Such a distinction is evidently essential if (1.4) is to be replaced by (1.5).

Before turning to the proof of the theorem let us consider an example which shows that (1.4) is about right in its dependence on $\max \left(\rho_{0},\left|a_{1}\right|\right)$. The details of the required estimates may be found in $\S 6$. Let $G(z)=(z-1)^{\alpha} F(z)$, where $\alpha=[2 \sigma]+1$ and

$$
F(z)=\prod_{k=1}^{\infty}\left(1-z e^{-k / 2 \sigma}\right)
$$

$G(z)$ satisfies (1.3) for $r \geqslant \rho_{0}$, for some $\rho_{0}$ satisfying $0<\rho_{0}<1$, and the first nonzero zero for $G$ is 1 . Moreover,

$$
\sigma(\log r)^{2}+O(1) \leqslant \log M(r, F) \leqslant \sigma(\log r)^{2}+\log r+O(1) .
$$


We therefore have that, for any $c>0, G(c z)$ satisfies (1.3) for $r>\rho_{0} / c$, has $1 / c$ as its first nonzero zero, and

$$
\begin{aligned}
(2 \sigma \log c+[2 \sigma]+1) \log r+O(1) & \leqslant \log M(r, G(c z))-\sigma(\log r)^{2} \\
& \leqslant(2 \sigma \log c+[2 \sigma]+2) \log r+O(1) .
\end{aligned}
$$

Hence

$$
\begin{aligned}
-2 \sigma\left(\log \left(\max \left(\rho_{0} / c, 1 / c\right)\right)-1\right) & \leqslant \frac{\lim }{r \rightarrow \infty} \frac{\log M(r, G(c z))-\sigma(\log r)^{2}}{\log r} \\
& \leqslant-2 \sigma\left(\log \left(\max \left(\rho_{0} / c, 1 / c\right)\right)-1\right)+2 .
\end{aligned}
$$

2. An auxiliary function. Let $n(t)=n(t, f)$ be the number of zeros of $f$ in $|z|<t$. We shall assume (without loss of generality) that $f(0)=1$, so that $n(t)=0$ for small values of $t$. We write

$$
u(z)=\log |f(z)|, \quad A(r, u)=\log m(r, f), \quad B(r, u)=\log M(r, f),
$$

and, given any $R>0$, introduce (cf. [5])

$$
\begin{aligned}
u^{*}(z, R)= & \int_{0}^{R} \log \left|\frac{R(t+z)}{R^{2}+z t}\right| d n(t) \\
& +B(R, u)-\left\{B(R, u)-\int_{0}^{R} n(t) \frac{d t}{t}\right\} \frac{R^{2}-|z|^{2}}{|R+z|^{2}} .
\end{aligned}
$$

$u^{*}$ is subharmonic in $|z|<R$, harmonic off the negative real axis and satisfies

$$
A\left(r, u^{*}\right)=u^{*}(-r, R) \leqslant A(r, u) \leqslant B(r, u) \leqslant u^{*}(r, R)=B\left(r, u^{*}\right),
$$

for $0 \leqslant r<R$. We choose $R^{\prime} \in(0, R)$ such that $n\left(R^{\prime}\right)=n(R)$ and such that

$$
n_{0}=\frac{B(R, u)-\int_{0}^{R} n(t) \frac{d t}{t}}{\log \left(R / R^{\prime}\right)}>0
$$

is an integer (which is possible since $n\left(R^{\prime}\right)=n(R)$ for all $R^{\prime}$ for which $R-R^{\prime}$ is small and positive, and the expression in the middle of (2.4) tends to $+\infty$ continuously as $R^{\prime} \rightarrow R-$ ), and define

$$
n_{1}(t)= \begin{cases}0, & 0 \leqslant t \leqslant \rho_{0}, \\ n(t), & \rho_{0}<t \leqslant R^{\prime} \\ n(t)+n_{0}, & R^{\prime}<t<R\end{cases}
$$

Set

$$
u_{1}^{*}(z, R)=\int_{0}^{R} \log \left|\frac{R(t+z)}{R^{2}+z t}\right| d n_{1}(t)+B(R, u) .
$$

Then $u_{1}^{*}(z, R)$ is subharmonic in $|z|<R$ and harmonic off the negative real axis. Also

LEMMA 1 . (a) $u_{1}^{*}(r, R) \geqslant u^{*}(r, R) \geqslant B(r, u)$, for $0 \leqslant r<R$.

(b) $u_{1}^{*}(r, R)-u_{1}^{*}(-r, R) \geqslant u^{*}(r, R)-u^{*}(-r, R) \geqslant-\log C(\sigma)$, for $\rho_{0}<r<R^{\prime}$.

(c) $u_{1}^{*}\left(R e^{i \theta}, R\right)=B(R, u)=\log M(R, f)$, for $-\pi<\theta<\pi$. 
Proof. Part (c) is evident. For part (a) we have

$$
\begin{aligned}
u_{1}^{*}(r, R)= & \int_{0}^{R} \log \left(\frac{R(t+r)}{R^{2}+r t}\right) d n(t)+n_{0} \log \left(\frac{R\left(R^{\prime}+r\right)}{R^{2}+r R^{\prime}}\right) \\
& +\left\{n\left(\rho_{0}\right) \log \left(\frac{R\left(\rho_{0}+r\right)}{R^{2}+r \rho_{0}}\right)-\int_{0}^{\rho_{0}} \log \left(\frac{R(t+r)}{R^{2}+r t}\right) d n(t)\right\} \\
& +B(R, u) \\
\geqslant & \int_{0}^{R} \log \left(\frac{R(t+r)}{R^{2}+r t}\right) d n(t)+n_{0} \log \left(\frac{R\left(R^{\prime}+r\right)}{R^{2}+r R^{\prime}}\right)+B(R, u) \\
= & u^{*}(r, R)+\frac{R-r}{R+r}\left\{B(R, u)-\int_{0}^{R} n(t) \frac{d t}{t}\right\}+n_{0} \log \left(\frac{R\left(R^{\prime}+r\right)}{R^{2}+r R^{\prime}}\right) \\
= & u^{*}(r, R)+n_{0}\left\{\frac{R-r}{R+r} \log \left(\frac{R}{R^{\prime}}\right)+\log \left(\frac{R\left(R^{\prime}+r\right)}{R^{2}+r R^{\prime}}\right)\right\} .
\end{aligned}
$$

The bracketed expression in the last line of (2.7), considered for the moment as a function of $R^{\prime}$, has derivative

$$
-\frac{r\left(R-R^{\prime}\right)^{2}(R-r)}{R^{\prime}(R+r)\left(R^{\prime}+r\right)\left(R^{2}+r R^{\prime}\right)} \leqslant 0 .
$$

The same bracketed expression vanishes when $R^{\prime}=R$, and it is therefore nonnegative for any $r \in[0, R), R^{\prime} \in(0, R)$. This proves part (a).

For part (b) we have, for $\rho_{0}<r<R$,

$$
\begin{aligned}
& u_{1}^{*}(r, R)-u_{1}^{*}(-r, R)= \int_{0}^{R} \log \left(\frac{R(t+r)}{R^{2}+r t}\right) d n(t)-\int_{0}^{R} \log \left|\frac{R(t-r)}{R^{2}-r t}\right| d n(t) \\
&+n_{0}\left\{\log \left(\frac{R\left(R^{\prime}+r\right)}{R^{2}+r R^{\prime}}\right)-\log \left|\frac{R\left(R^{\prime}-r\right)}{R^{2}-r R^{\prime}}\right|\right\} \\
&+n\left(\rho_{0}\right)\left\{\log \left(\frac{R\left(\rho_{0}+r\right)}{R^{2}+r \rho_{0}}\right)-\log \left|\frac{R\left(\rho_{0}-r\right)}{R^{2}-r \rho_{0}}\right|\right\} \\
&-\left\{\int_{0}^{\rho_{0}} \log \left(\frac{R(t+r)}{R^{2}+r t}\right) d n(t)-\int_{0}^{\rho_{0}} \log \left|\frac{R(t-r)}{R^{2}-r t}\right| d n(t)\right\} \\
& \geqslant \int_{0}^{R} \log \left(\frac{R(t+r)}{R^{2}+r t}\right) d n(t)-\int_{0}^{R} \log \left|\frac{R(t-r)}{R^{2}-r t}\right| d n(t) \\
&+n_{0}\left\{\log \left(\frac{R\left(R^{\prime}+r\right)}{R^{2}+r R^{\prime}}\right)-\log \left|\frac{R\left(R^{\prime}-r\right)}{R^{2}-r R^{\prime}}\right|\right\} \\
&= u^{*}(r, R)-u^{*}(-r, R) \\
&+n_{0}\left\{\log \left(\frac{R\left(R^{\prime}+r\right)}{R^{2}+r R^{\prime}}\right)-\log \left|\frac{R\left(R^{\prime}-r\right)}{R^{2}-r R^{\prime}}\right|\right. \\
& \\
&\left.\left.\qquad \begin{array}{ll}
R \\
R^{\prime}
\end{array}\right)\left[\frac{R+r}{R-r}-\frac{R-r}{R+r}\right]\right\} .
\end{aligned}
$$


In order to prove part (b) of Lemma 1, then, it is sufficient to show that the bracketed expression in the last line of (2.8) is nonnegative for $\rho_{0}<r<R^{\prime}$. To this end consider the same expression as a function of $R^{\prime}$ on $(r, R)$, for any $r \in[0, R)$. Its derivative is

$$
\begin{gathered}
\frac{1}{R^{\prime}+r}-\frac{r}{R^{2}+r R^{\prime}}-\frac{1}{R^{\prime}-r}-\frac{r}{R^{2}-r R^{\prime}}+\frac{1}{R^{\prime}}\left\{\frac{R+r}{R-r}-\frac{R-r}{R+r}\right\} \\
\quad=-\frac{2 r\left(R-R^{\prime}\right)^{2}\left\{R^{\prime}\left(R^{2}+r^{2}\right)^{2}+2 r^{2} R\left(R^{2}+R^{\prime 2}\right)\right\}}{R^{\prime}\left(R^{\prime 2}-r^{2}\right)\left(R^{4}-r^{2} R^{\prime 2}\right)\left(R^{2}-r^{2}\right)} \\
\leqslant 0
\end{gathered}
$$

for $0 \leqslant r<R^{\prime}<R$. But the bracketed expression vanishes at $R^{\prime}=R$ and so is nonnegative for $R^{\prime} \in(r, R)$. This proves part (b) of the lemma.

3. Rearranging the zeros. Here and elsewhere we shall speak of the "zeros" of $u_{1}^{*}(r, R)$, which are to be understood as the unit point masses associated with the counting function $n_{1}(t)$. Let these zeros be $r_{1} \leqslant r_{2} \leqslant \cdots \leqslant r_{N}$, so that $r_{1} \geqslant \rho_{0}$, $r_{N}=R^{\prime}$ and $N=n(R)+n_{0}$. From part (b) of Lemma 1 we have

$$
\begin{gathered}
-\log C(\sigma) \leqslant u_{1}^{*}(r, R)-u_{1}^{*}(-r, R)=\int_{0}^{R} \log \left|\frac{t+r}{t-r} \cdot \frac{R^{2}-r t}{R^{2}+r t}\right| d n_{1}^{*}(t) \\
=\int_{0}^{R} \log \left|\frac{T(r)+T(t)}{T(r)-T(t)}\right| d n_{1}^{*}(t)=\sum_{k=1}^{N} \log \left|\frac{T(r)-T\left(r_{k}\right)}{T(r)-T\left(r_{k}\right)}\right|,
\end{gathered}
$$

where

$$
T(r)=\frac{A r}{R^{2}-r^{2}},
$$

and $A$ is a constant chosen so that $T\left(r_{1}\right)=1$. Let us observe that $T\left(r_{N}\right) \leqslant e^{(N-1) / 2 \sigma}$. For in [3] it is shown that the largest value of the minimum of

$$
\sum_{k=1}^{N} \log \left|\frac{T(r)+T\left(r_{k}\right)}{T(r)-T\left(r_{k}\right)}\right|
$$

for $r_{1}<r<r_{N}$, no matter how $r_{2}, \ldots, r_{N-1}$ are distributed in $\left[r_{1}, r_{N}\right]$, is less than

$$
\log \prod_{k=1}^{\infty}\left\{\frac{1+\exp (-(2 k-1) \tau / 2)}{1-\exp (-(2 k-1) \tau / 2)}\right\}^{2}
$$

where

$$
\tau=\frac{\log T\left(r_{N}\right)-\log T\left(r_{1}\right)}{N-1}=\frac{\log T\left(r_{N}\right)}{N-1} .
$$

If $T\left(r_{N}\right)>e^{(N-1) / 2 \sigma}$ then $\tau>\frac{1}{2 \sigma}$ and so the number (3.3) is $-\log C\left(\frac{1}{2 \tau}\right)<$ $-\log C(\sigma)$. But this contradicts (3.1) so we must have $T\left(r_{N}\right) \leqslant e^{(N-1) / 2 \sigma}$. 
Write $r_{k}=T^{-1}\left(e^{(k-1) / 2 \sigma}\right)$ for $k>N$ and for $k<1$. Then, from (3.1),

$$
\sum_{k=-\infty}^{\infty} \log \left|\frac{T(r)+T\left(r_{k}\right)}{T(r)-T\left(r_{k}\right)}\right|>-\log C(\sigma),
$$

for $r_{1}<r<r_{N}$. The plan is now to fix any $\rho, 0<\rho<R$ (the precise choice of which turns out to be immaterial) and to rearrange $r_{1}, r_{2}, \ldots, r_{N}$ within $\left[r_{1}, T^{-1}\left(e^{(N-1) / 2 \sigma}\right)\right]$ in such a way that $u_{1}^{*}(\rho, R)$ is maximized while

$$
r_{1} \text { remains fixed, }
$$

and

$$
\sum_{k=-\infty}^{\infty} \log \left|\frac{T(r)+T\left(r_{k}\right)}{T(r)-T\left(r_{k}\right)}\right| \geqslant-\log C(\sigma) \quad \text { for } r_{1}<r<r_{N} .
$$

Let $r_{1}^{\prime}=r_{1} \leqslant r_{2}^{\prime} \leqslant \cdots \leqslant r_{N}^{\prime}$ be the positions of the rearranged zeros which maximize $u_{1}^{*}(\rho, R)$ subject to (3.4) and (3.5), and let us write $r_{k}^{\prime}=r_{k}$ for $k<1$ and for $k>N$. Then we have

LEMMA 2. There are two possibilities. Either

(i) there exists an integer $M$ such that $1<M \leqslant N-1$ and $r_{1}^{\prime}=r_{1}<r_{2}^{\prime}<\cdots<r_{M}^{\prime}$ $<r_{M+1}^{\prime}=r_{M+2}^{\prime}=\cdots=r_{N}^{\prime}=T^{-1}\left(e^{(N-1) / 2 \sigma}\right)$. Further, if for $1 \leqslant p \leqslant N-1$,

$$
I_{p}= \begin{cases}\min _{r_{p}^{\prime}<r<r_{p+1}^{\prime}}\left\{\sum_{k=-\infty}^{\infty} \log \mid \frac{T(r)+T\left(r_{k}^{\prime}\right)}{\left.T(r)-T\left(r_{k}^{\prime}\right) \mid\right\}}\right. & \text { if } r_{p}^{\prime}<r_{p+1}^{\prime}, \\ +\infty & \text { if } r_{p}^{\prime}=r_{p+1}^{\prime},\end{cases}
$$

then $I_{p}=-\log C(\sigma)$, for $p=1,2, \ldots, M-1, I_{M} \geqslant-\log C(\sigma)$ and $I_{p}=+\infty$, for $p=M+1, \ldots, N-1 . O r$,

(ii) $r_{1}^{\prime}<r_{2}^{\prime}<\cdots<r_{N-1}^{\prime}<r_{N}^{\prime}<T^{-1}\left(e^{(N-1) / 2 \sigma}\right)$ and $I_{p}=-\log C(\sigma), p=$ $1,2, \ldots, N-1$.

Lemma 2 follows from

Lemma 3. (a) Suppose that $r_{1}^{\prime}<r_{2}^{\prime}<r_{3}^{\prime} \leqslant r_{4}^{\prime} \leqslant \cdots \leqslant r_{N}^{\prime}$. Then shifting $r_{2}^{\prime}$ slightly to the right within $\left(r_{1}^{\prime}, r_{3}^{\prime}\right)$ increases $I_{p}$ for $p>1$, decreases $I_{1}$ and increases $u_{1}^{*}(\rho, R)$.

(b) Suppose that $r_{1}^{\prime}=r_{2}^{\prime}=\cdots=r_{j}^{\prime}<r_{j+1}^{\prime} \leqslant \cdots \leqslant r_{N}^{\prime}$. Then the small shift of $r_{j}^{\prime}$ to the right within $\left(r_{1}^{\prime}, r_{j+1}^{\prime}\right)$ increases $I_{p}$ for $j \leqslant p \leqslant N-1$, decreases $I_{j-1}$, leaves $I_{1}, I_{2}, \ldots, I_{j-2}$ the same and increases $u_{1}^{*}(\rho, R)$.

(c) If $r_{1}^{\prime}<r_{m}^{\prime} \leqslant r_{m+1}^{\prime}<r_{N}^{\prime}$ then, for small values of $k>1$, the shift $r_{m}^{\prime} \rightarrow k^{-1} r_{m}^{\prime}$ and $r_{m+1}^{\prime} \rightarrow k r_{m+1}^{\prime}$ increases $I_{p}$ for $p \neq m$, decreases $I_{m}$ and increases $u_{1}^{*}(\rho, R)$.

Before proving Lemma 3 let us show that Lemma 2 follows from Lemma 3. We cannot have $r_{1}^{\prime}<r_{m}^{\prime}=r_{m+1}^{\prime}<r_{N}^{\prime}$, from part (c) of Lemma 3, and we cannot have $r_{1}^{\prime}=r_{2}^{\prime}$, from part (b). Thus the points $r_{k}^{\prime}$ must be distributed in either one of the ways described in the two parts of Lemma 2. Concerning the values of $I_{p}$, suppose that $I_{1}>-\log C(\sigma)$. Then from part (a) of Lemma 3, a small shift of $r_{2}^{\prime}$ to the right will increase $u_{1}^{*}(\rho, R)$ while keeping $I_{p} \geqslant-\log C(\sigma)$, for $p>1$, and also $I_{1}>$ $-\log C(\sigma)$ if the shift of $r_{2}^{\prime}$ is small enough. But this contradicts the definition of 
$r_{1}^{\prime}, \ldots, r_{N}^{\prime}$, so we must have $I_{1}=-\log C(\sigma)$. Now suppose that $I_{q}>-\log C(\sigma)$ for some $q>1$, where $q \leqslant M-1$ in case (i). Then the shift $r_{q}^{\prime} \rightarrow k^{-1} r_{q}^{\prime}, r_{q+1}^{\prime} \rightarrow k r_{q+1}^{\prime}$ decreases $I_{q}$ (which may be kept greater than $-\log C(\sigma)$ if $k>1$ is close enough to $1)$, keeps $I_{p} \geqslant-\log C(\sigma)$ for $p \neq q$, and increases $u_{1}^{*}(\rho, R)$. This again contradicts the definition of $r_{1}^{\prime}, r_{2}^{\prime}, \ldots, r_{N}^{\prime}$, and so $I_{q}>-\log C(\sigma)$ can arise only when $q \geqslant M$ in case (i) of Lemma 2. This proves Lemma 2.

It remains to prove Lemma 3 and we consider the parts in turn. For part (a), if $r>r_{2}^{\prime}$ then $T(r)>T\left(r_{2}^{\prime}\right)$ and so

$$
\sum_{k=-\infty}^{\infty} \log \left|\frac{T(r)+T\left(r_{k}^{\prime}\right)}{T(r)-T\left(r_{k}^{\prime}\right)}\right|=\sum_{\substack{k=-\infty \\ k \neq 2}}^{\infty} \log \left|\frac{T(r)+T\left(r_{k}^{\prime}\right)}{T(r)-T\left(r_{k}^{\prime}\right)}\right|+\log \left(\frac{T(r)+T\left(r_{2}^{\prime}\right)}{T(r)-T\left(r_{2}^{\prime}\right)}\right),
$$

which is increased if $r_{2}^{\prime}$ is slightly increased since $(a+x) /(a-x)$ is an increasing function of $x$ for $x \neq a$ and $T(x)$ is increasing on $(0, R)$. Thus $I_{p}$ increases for $p>1$. Similarly, for $r_{1}^{\prime}<r<r_{2}^{\prime}$, we have $T(r)<T\left(r_{2}^{\prime}\right)$ so

$$
\sum_{k=-\infty}^{\infty} \log \left|\frac{T(r)+T\left(r_{k}^{\prime}\right)}{T(r)-T\left(r_{k}^{\prime}\right)}\right|=\sum_{\substack{k=-\infty \\ k \neq 2}}^{\infty} \log \left|\frac{T(r)+T\left(r_{k}^{\prime}\right)}{T(r)-T\left(r_{k}^{\prime}\right)}\right|+\log \left(\frac{T\left(r_{2}^{\prime}\right)+T(r)}{T\left(r_{2}^{\prime}\right)-T(r)}\right),
$$

which is decreased if $r_{2}^{\prime}$ is shifted slightly to the right since $(x+a) /(x-a)$ is decreasing in $x$ for $x \neq a$ and $T(x)$ is increasing on $(0, R)$. Thus $I_{1}$ decreases. Finally, the effect on $u_{1}^{*}(\rho, R)$ of a slight shift of $r_{2}^{\prime}$ to the right is (if the final position is $r_{2}^{\prime \prime}$ )

$$
\log \left(\frac{R\left(\rho+r_{2}^{\prime \prime}\right)}{R^{2}+r_{2}^{\prime \prime} \rho}\right)-\log \left(\frac{R\left(\rho+r_{2}^{\prime}\right)}{R^{2}+r_{2}^{\prime} \rho}\right)>0
$$

since

$$
\frac{d}{d x}\left(\frac{R(\rho+x)}{R^{2}+\rho x}\right)=\frac{R\left(R^{2}-\rho^{2}\right)}{\left(R^{2}+\rho x\right)^{2}}>0 .
$$

For part (b) observe that $I_{j-1}$ certainly decreases (since it changes from $+\infty$ to a finite value) and $I_{1}, I_{2}, \ldots, I_{j-2}$ all remain $+\infty$. The considerations made in the proof of part (a) show that $I_{p}$ increases for $p \geqslant j$ and that $u_{1}^{*}(\rho, R)$ increases.

Finally we prove part (c). The shift described in part (c) produces a change in

$$
\sum_{k=-\infty}^{\infty} \log \left|\frac{T(r)+T\left(r_{k}^{\prime}\right)}{T(r)-T\left(r_{k}^{\prime}\right)}\right|
$$

given by $h(k)-h(1)$, where

$$
h(k)=\log \left|\frac{r+k^{-1} r_{m}^{\prime}}{R^{2}+k^{-1} r_{m}^{\prime} r} \cdot \frac{R^{2}-k^{-1} r_{m}^{\prime} r}{r-k^{-1} r_{m}^{\prime}}\right|+\log \left|\frac{r+k r_{m+1}^{\prime}}{R^{2}+k r_{m+1}^{\prime} r} \cdot \frac{R^{2}-k r_{m+1}^{\prime} r}{r-k r_{m+1}^{\prime}}\right| .
$$

After a certain amount of simplification we obtain $h^{\prime}(k)=X / Y$, where

$$
\begin{aligned}
X= & 2 r k^{-1}\left(R^{2}-r^{2}\right)\left(k r_{m+1}^{\prime}-k^{-1} r_{m}^{\prime}\right)\left(R^{2}-r_{m}^{\prime} r_{m+1}^{\prime}\right) \\
& \cdot\left\{\left(R^{4}+r^{2} r_{m}^{\prime} r_{m+1}^{\prime}\right)\left(r^{2}+r_{m}^{\prime} r_{m+1}^{\prime}\right)+R^{2} r^{2}\left(k r_{m+1}^{\prime}+k^{-1} r_{m}^{\prime}\right)^{2}\right\}
\end{aligned}
$$


and

$$
Y=\left(r^{2}-k^{2} r_{m+1}^{\prime 2}\right)\left(r^{2}-k^{-2} r_{m}^{\prime 2}\right)\left(R^{4}-r^{2} k^{2} r_{m+1}^{\prime 2}\right)\left(R^{4}-r^{2} k^{-2} r_{m}^{\prime 2}\right) .
$$

Thus, for small $k>1, h^{\prime}(k)<0$ if $r_{m}^{\prime}<r<r_{m+1}^{\prime}$, while $h^{\prime}(k)>0$ if $r>k r_{m+1}^{\prime}$ or if $r<k^{-1} r_{m}^{\prime}$. Thus, for small values of $k>1$, the described shift increases (3.7) if $r>k r_{m+1}^{\prime}$ and if $r<k^{-1} r_{m}^{\prime}$ (and so increases $I_{p}$ for $p \neq m$ ) and decreases (3.4) if $r_{m}^{\prime}<r<r_{m+1}^{\prime}$ (and so decreases $I_{m}$ ). This proves part of part (c) of Lemma 3.

For the second part of part (c), the shift described in the lemma alters $u_{1}^{*}(\rho, R)$ by $g(k)-g(1)$, where

Now for $k>1$,

$$
g(k)=\log \left(\frac{R\left(\rho+k^{-1} r_{m}^{\prime}\right)}{R^{2}+k^{-1} r_{m}^{\prime} \rho}\right)+\log \left(\frac{R\left(\rho+k r_{m+1}^{\prime}\right)}{R^{2}+k r_{m+1}^{\prime} \rho}\right) .
$$

$$
\begin{gathered}
g^{\prime}(k)=k^{-1}\left(R^{2}-\rho^{2}\right)\left\{\frac{k r_{m+1}^{\prime}}{\left(\rho+k r_{m+1}^{\prime}\right)\left(R^{2}+\rho k r_{m+1}^{\prime}\right)}\right. \\
\left.-\frac{k^{-1} r_{m}^{\prime}}{\left(\rho+k^{-1} r_{m}^{\prime}\right)\left(R^{2}+\rho k^{-1} r_{m}^{\prime}\right)}\right\}>0,
\end{gathered}
$$

since $\frac{d}{d x}\left\{\frac{1}{x}(\rho+x)\left(R^{2}+\rho x\right)\right\}=\frac{\rho}{x^{2}}\left(x^{2}-R^{2}\right)<0$ for $0<x<R$. Thus $g(k)$ is increasing for $k>1$ and it follows that $g(k)-g(1)>0$ for small $k>1$, i.e., $u_{1}^{*}(\rho, R)$ increases for small $k>1$. This completes the proof of Lemma 3 .

4. The positions of the zeros. Lemma 2 provides a description of the positions of the zeros at which $u_{1}^{*}(\rho, R)$ is maximized, for any $\rho \in(0, R)$. We now analyze the situation a little further and show that $r_{k}^{\prime}=T^{-1}\left(e^{(k-1) / 2 \sigma}\right), k=1,2, \ldots, N$. Once this is done $u_{1}^{*}(\rho, R)$ may be calculated directly.

Now, exactly as in [3],

$$
H(r)=\sum_{k=-\infty}^{\infty} \log \left|\frac{T(r)+T\left(r_{k}^{\prime}\right)}{T(r)-T\left(r_{k}^{\prime}\right)}\right|=2 \int_{0}^{\infty} \nu_{r}(t) \frac{e^{t}}{e^{2 t}-1} d t,
$$

where $\nu_{r}(t)$ is the number of points $\log T\left(r_{k}^{\prime}\right)$ contained in $(\log T(r)-t, \log T(r)+t)$. According to Lemma 2 there are three possible graphs for $H(r)$, one corresponding to part (i) of Lemma 2, and two corresponding to part (ii). In all cases we consider the graph only over a selected interval.

For part (i), see Figure 1. (There is also the possibility that the minimum in the last interval is exactly $-\log C(\sigma)$.)

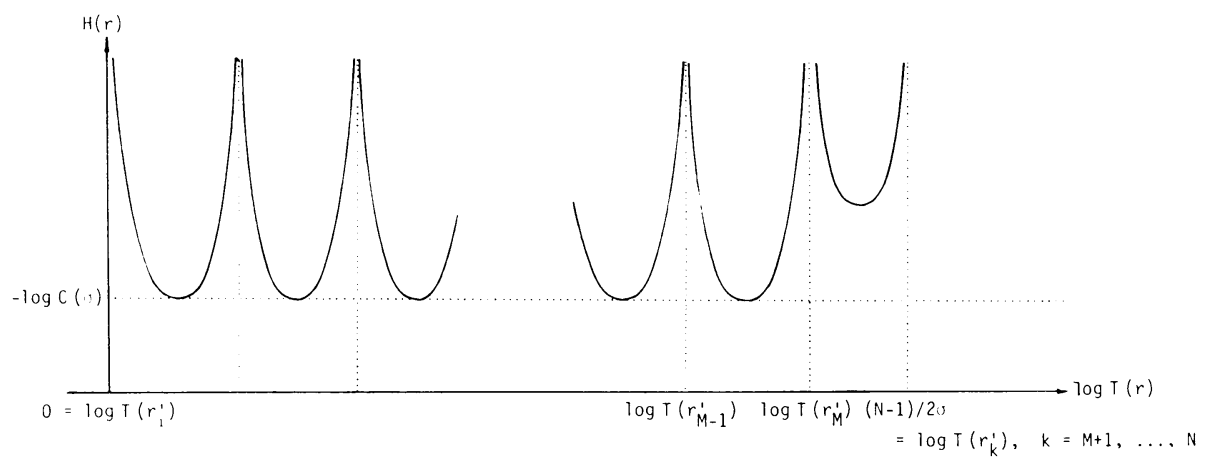

Figure 1 


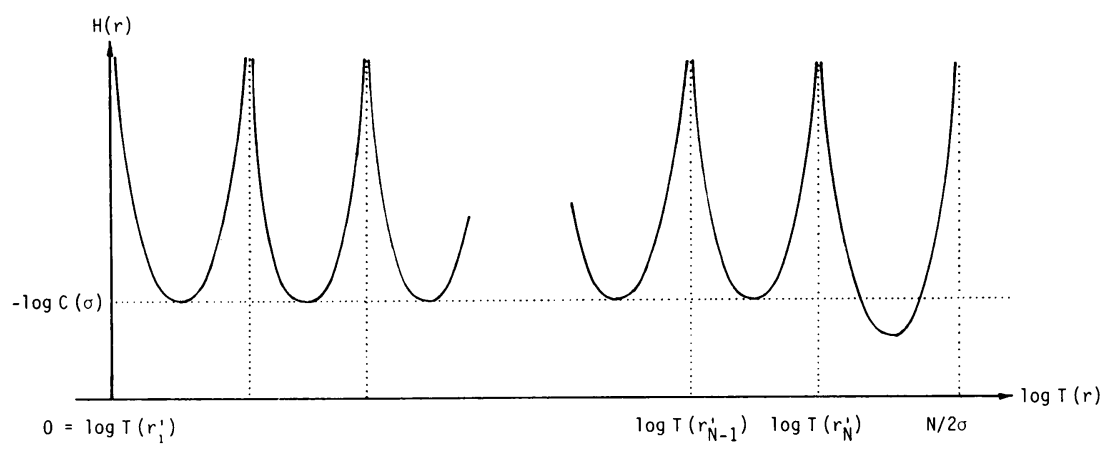

FIGURE 2

For part (ii), either refer to Figure 2 or to a graph the same except that on the interval $\left(\log T\left(r_{N}^{\prime}\right), N / 2 \sigma\right)$ the minimum is $\geqslant-\log C(\sigma)$. We shall show now that the disposition of the zeros giving rise to the first graph is impossible, and that the only disposition of the zeros corresponding to the second graph is in fact the one in which all minima are $-\log C(\sigma)$.

From (4.1) we see that the contribution of the zero $r_{j}^{\prime}$ to $H(r)$ is

$$
2 \int_{\left|\log \left(T(r) / T\left(r_{j}^{\prime}\right)\right)\right|}^{\infty} \frac{e^{t}}{e^{2 t}-1} d t
$$

which is increased if $r_{j}^{\prime}$ is moved towards $r$ and decreased if $r_{j}^{\prime}$ is moved away from $r$. Further, the amount of the increase or decrease is smaller the further removed $r$ is from $r_{j}^{\prime}$. Thus, for the first graph, by shifting $T\left(r_{M+1}^{\prime}\right), \ldots, T\left(r_{N-1}^{\prime}\right)$ slightly to the left and separating them (while preserving their order) we shall produce a new graph of the form shown in Figure 3.

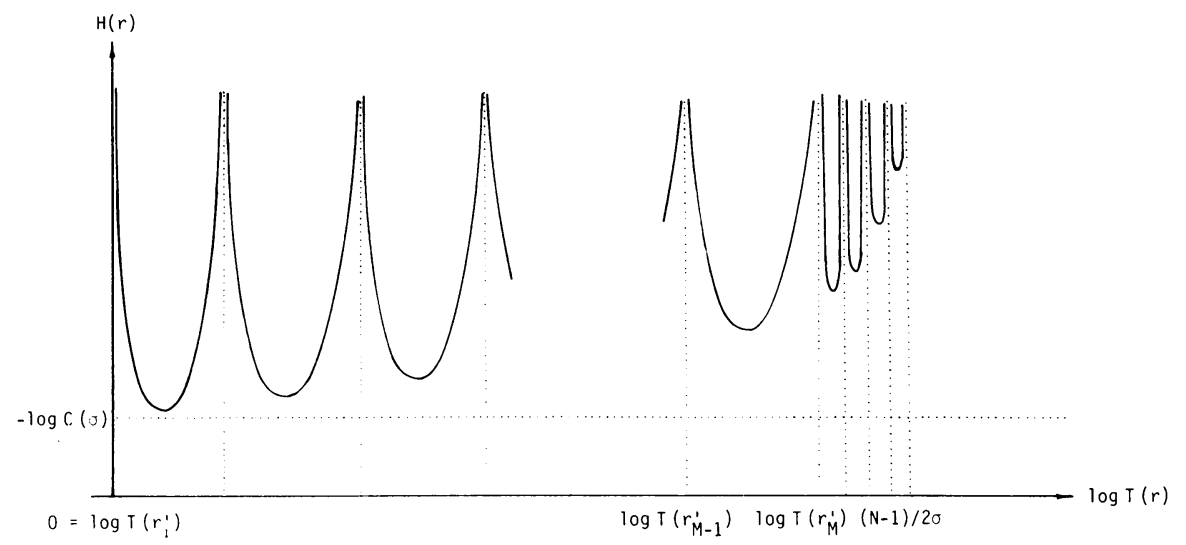

FigURE 3 
Now following the procedure described in [3] of "cycling" the points $T\left(r_{2}^{\prime}\right), \ldots, T\left(r_{N-1}^{\prime}\right)$, we shall produce a graph in which all minima are the same, with a value strictly greater than $-\log C(\sigma)$. But from the lemma of [3] the fact that all minima are the same implies that $T\left(r_{j}^{\prime}\right)=e^{(j-1) / 2 \sigma}$, which gives the exact value of the minima as $-\log C(\sigma)$. This contradiction discounts the possibility of the first graph.

In the case of the graphs corresponding to part (ii), if the minimum on the last interval is not $-\log C(\sigma)$, a cycling of the points $T\left(r_{2}^{\prime}\right), \ldots, T\left(r_{N-1}^{\prime}\right)$ will produce a graph in which all minima are the same, with a value strictly greater or strictly less than $-\log C(\sigma)$. This again leads to a contradiction.

We conclude that all the points $\log T\left(r_{1}^{\prime}\right), \ldots, \log T\left(r_{N}^{\prime}\right)$ are separated and that all the minima are equal. From the lemma of [3] it follows that $\log T\left(r_{j}^{\prime}\right)=$ $(j-1) / 2 \sigma, j=1,2, \ldots, N$.

5. Completion of the proof of the theorem. We have $r_{j}^{\prime}=T^{-1}\left(e^{(j-1) / 2 \sigma}\right)$ and from part (a) of Lemma 1,

$$
\begin{aligned}
B(r, u) & \leqslant u^{*}(r, R)=B(R, u)+\int_{0}^{R} \log \left(\frac{R(t+r)}{R^{2}+r t}\right) d n_{1}(t) \\
& =B(R, u)-\int_{0}^{R} n_{1}(t)\left\{\frac{1}{t+r}-\frac{r}{R^{2}+r t}\right\} d t,
\end{aligned}
$$

for $0 \leqslant r<R$. Also, for $r_{j}^{\prime}<t<r_{j+1}^{\prime}$,

$$
n_{1}(t)=j=2 \sigma \log T\left(r_{j+1}^{\prime}\right)>2 \sigma \log T(t)=2 \sigma \log \left(\frac{A t}{\left(R^{2}-t^{2}\right)}\right),
$$

while $n_{1}(t)=0$ for $0 \leqslant t<r_{1}^{\prime}$. Now the number $n_{0}$ of (2.1) may be made arbitrarily large by taking $R^{\prime}$ arbitrarily close to $R$, and this enables us to take $N$ arbitrarily large. From (5.1), then, since $r_{1}^{\prime}=r_{1}$,

$$
\begin{aligned}
B(r, u) & \leqslant B(R, u)-2 \sigma \int_{r_{1}}^{R} \log \left(\frac{A t}{R^{2}-t^{2}}\right)\left\{\frac{1}{t+r}-\frac{r}{R^{2}+r t}\right\} d t \\
& =B(R, u)-2 \sigma\left\{I_{1}+I_{2}+I_{3}\right\}
\end{aligned}
$$

where

$$
\begin{gathered}
I_{1}=\log A \int_{r_{1}}^{R}\left\{\frac{1}{t+r}-\frac{r}{R^{2}+r t}\right\} d t=-\log A \log \left(\frac{R\left(r_{1}+r\right)}{R^{2}+r r_{1}}\right), \\
I_{2}=\int_{r_{1}}^{R} \log t\left\{\frac{1}{t+r}-\frac{r}{R^{2}+r t}\right\} d t
\end{gathered}
$$

and

$$
I_{3}=-\int_{r_{1}}^{R} \log \left(R^{2}-t^{2}\right)\left\{\frac{1}{t+r}-\frac{r}{R^{2}+r t}\right\} d t .
$$


We estimate $I_{2}$ and $I_{3}$ in turn. For $r$ fixed, $r>\max \left(1, r_{1}\right)$ and $R>2 r$,

$$
\begin{aligned}
I_{2}= & \int_{r_{1}}^{R} \frac{\log t}{t+r} d t+O\left(\frac{r}{R} \log R\right) \\
= & \int_{r_{1} / r}^{R / r} \frac{\log t+\log r}{t+1} d t+O\left(\frac{r}{R} \log R\right) \\
= & \int_{r_{1} / r}^{R / r} \frac{\log t}{t+1} d t+\log r \log \left(\frac{R+r}{r+r_{1}}\right)+O\left(\frac{r}{R} \log R\right) \\
(5.6)= & \int_{r_{1} / r}^{R / r} \frac{t}{t+1} \frac{d}{d t}\left(\frac{1}{2}(\log t)^{2}\right) d t+\log r \log \left(\frac{R+r}{r+r_{1}}\right)+O\left(\frac{r}{R} \log R\right) \\
= & \frac{1}{2} \frac{R}{R+r}\left(\log \frac{R}{r}\right)^{2}-\frac{1}{2} \frac{r_{1}}{r_{1}+r}\left(\log \frac{r_{1}}{r}\right)^{2}-\frac{1}{2} \int_{r_{1} / r}^{R / r}(\log t)^{2} \frac{1}{(t+1)^{2}} d t \\
& +\log r \log \left(\frac{R+r}{r+r_{1}}\right)+O\left(\frac{r}{R} \log R\right) \\
= & \frac{1}{2}\left(\log \frac{R}{r}\right)^{2}+\log r \log R+O(1) \\
= & \frac{1}{2}(\log R)^{2}+O(1)
\end{aligned}
$$

as $R \rightarrow \infty$. Also, as $R \rightarrow \infty$,

$$
\begin{aligned}
I_{3} & =-\int_{r_{1}}^{R} \frac{\log \left(R^{2}-t^{2}\right)}{t+r} d t+O\left(\frac{\log R}{R}\right) \\
& =-R \int_{r_{1} / R}^{1} \frac{2 \log R+\log \left(1-t^{2}\right)}{R t+r} d t+O\left(\frac{\log R}{R}\right) \\
& =2 \log R \log \left(\frac{r+r_{1}}{r+R}\right)-R \int_{r_{1} / R}^{1} \frac{\log \left(1-t^{2}\right)}{R t+r} d t+O(1) \\
& \geqslant 2 \log R \log \left(\frac{r+r_{1}}{r+R}\right)+O(1) .
\end{aligned}
$$

Combining (5.2), (5.3), (5.6) and (5.7) we obtain, as $R \rightarrow \infty$,

$$
\begin{aligned}
B(r, u) \leqslant & B(R, u)-\sigma(\log R)^{2} \\
& -4 \sigma \log R \log \left(\frac{r+r_{1}}{r+R}\right)+2 \sigma \log A \log \left(\frac{R\left(r_{1}+r\right)}{R^{2}+r r_{1}}\right)+O(1) .
\end{aligned}
$$

Since $A$ was chosen so that $A r_{1} /\left(R^{2}-r_{1}^{2}\right)=1,(5.8)$ becomes

$$
\begin{aligned}
B(r, u) & \leqslant B(R, u)-\sigma(\log R)^{2} \\
& -4 \sigma \log R\left\{\log \left(r+r_{1}\right)-\log R+O\left(\frac{1}{R}\right)\right\} \\
& +2 \sigma\left\{2 \log R-\log r_{1}+O\left(\frac{1}{R^{2}}\right)\right\}\left\{-\log R+\log \left(r+r_{1}\right)+O\left(\frac{1}{R^{2}}\right)\right\} \\
& +O(1) \\
= & B(R, u)-\sigma(\log R)^{2}+2 \sigma \log r_{1} \log R+O(1)
\end{aligned}
$$


as $R \rightarrow \infty$. From the construction, $r_{1}=\max \left(\rho_{0},\left|a_{1}\right|\right)$, where $a_{1}$ is the first nonzero zero of $f$, and therefore

$$
B(r, u) \leqslant B(R, u)-\sigma(\log R)^{2}+2 \sigma \log R \log \left(\max \left(\rho_{0},\left|a_{1}\right|\right)\right)+O(1)
$$

as $R \rightarrow \infty$. Now, if the conclusion of the theorem is false then we may choose a sequence of $R \rightarrow \infty$ so that the right-hand side of (5.10) tends to $-\infty$, for any fixed $r$. Thus $B(r, u)=-\infty$ for every $r$ and this contradiction proves the theorem.

6. The example. It remains to provide some detail concerning the assertions made about the example in the first section of the paper. The estimates for $M(r, F)$ follow from

$$
\log M(r, F)=\int_{0}^{\infty} \frac{r n(t)}{t(t+r)} d t,
$$

where $n(t)$, the counting function for the zeros of $F$, satisfies $n(t)=0$, for $0 \leqslant t \leqslant 1$, and

$$
2 \sigma \log t \leqslant n(t) \leqslant 2 \sigma \log t+1,
$$

for $t>1$. To see that $G$ satisfies

$$
\log M(r, G)-\log m(r, G) \geqslant-\log C(\sigma)
$$

for $r \geqslant \rho_{0}$, where $0<\rho_{0}<1$, we begin with the formula (cf. [3])

$$
\log M\left(e^{r}, G\right)-\log m\left(e^{r}, G\right)=2 \int_{0}^{\infty} \nu_{r}(t) \frac{e^{t}}{e^{2 t}-1} d t,
$$

where $\nu_{r}(t)$ is the number of zeros of $G$ in $\left(e^{r-t}, e^{r+t}\right)$ i.e. if the zeros of $G$ are denoted by $r_{n}, \nu_{r}(t)$ is the number of points $\log r_{n}$ in $(r-t, r+t)$. Now, if $\eta_{r}(t)$ is the number of integer multiples of $1 / 2 \sigma$, positive, negative and zero, in $(r-t, r+t)$, then

$$
\begin{aligned}
I(r) & =\int_{0}^{\infty} \eta_{r}(t) \frac{e^{t}}{e^{2 t}-1} d t=\sum_{k=-\infty}^{\infty} \int_{|r-k / 2 \sigma|}^{\infty}\left\{\frac{e^{t}}{e^{t}-1}-\frac{e^{t}}{e^{t}+1}\right\} d t \\
& =\sum_{k=-\infty}^{\infty} \log \left(\frac{1-\exp (-|r-k / 2 \sigma|)}{1+\exp (-|r-k / 2 \sigma|)}\right) .
\end{aligned}
$$

The minimum value of $I(r)$ occurs at odd half-multiples of $1 / 2 \sigma$, and the minimum value is $-\log C(\sigma)$. We shall therefore have (6.1) for $r \geqslant \rho_{0}$, where $0<\rho_{0}<1$, if it can be shown that

$$
\int_{0}^{\infty} \nu_{r}(t) \frac{e^{t}}{e^{2 t}-1} d t-\int_{0}^{\infty} \eta_{r}(t) \frac{e^{t}}{e^{2 t}-1} d t \geqslant 0
$$

for $r \geqslant 0$. Taking account of the definition of the zeros of $G$ we see that the left-hand side of $(6.2)$ is

$$
\alpha \int_{r}^{\infty} \frac{e^{t}}{e^{2 t}-1} d t-\int_{0}^{\infty} \eta_{r}^{-}(t) \frac{e^{t}}{e^{2 t}-1} d t
$$


where $\eta_{r}^{-}(t)$ is the number of strictly negative multiples of $1 / 2 \sigma$ in $(r-t, r+t)$. We have

$$
\begin{aligned}
\int_{0}^{\infty} \eta_{r}^{-}(t) \frac{e^{t}}{e^{2 t}-1} d t & =\sum_{k=1}^{\infty} \int_{r+k / 2 \sigma}^{\infty} \frac{e^{-x}}{1-e^{-2 x}} d x=\sum_{k=1}^{\infty} \int_{r+k / 2 \sigma}^{\infty}\left\{\sum_{j=0}^{\infty} e^{-(2 j+1) x}\right\} d x \\
& =\sum_{j=0}^{\infty} \frac{e^{-(2 j+1) r}}{2 j+1} \sum_{k=1}^{\infty} e^{-(2 j+1) k / 2 \sigma}=\sum_{j=0}^{\infty} \frac{e^{-(2 j+1) r}}{2 j+1} \cdot \frac{1}{e^{(2 j+1) / 2 \sigma}-1} \\
& \leqslant 2 \sigma \sum_{j=0}^{\infty} \frac{e^{-(2 j+1) r}}{2 j+1}=2 \sigma \int_{r}^{\infty}\left\{\sum_{j=0}^{\infty} e^{-(2 j+1) x}\right\} d x=2 \sigma \int_{r}^{\infty} \frac{e^{t}}{e^{2 t}-1} d t .
\end{aligned}
$$

Thus (6.3) holds with $\alpha=[2 \sigma]+1$, and this completes the analysis of the example.

\section{REFERENCES}

1. P. D. Barry, The minimum modulus of small integral and subharmonic functions, Proc. London Math. Soc. (3) 12 (1962), 445-495.

2. P. C. Fenton, Regularity of certain small subharmonic functions, Trans. Amer. Math. Soc. 262 (1980), 473-486.

3. The minimum of small entire functions, Proc. Amer. Math. Soc. 81 (1981), 557-561.

4. A. A. Gol'dberg, Minimum modulus of a meromorphic function of slow growth, Mat. Zametki 25 (1979), 835-844; English transl. in Math. Notes (1979), 432-437.

5. Ulf Hellsten, Bo Kjellberg and Folke Norstad, Subharmonic functions in a circle, Ark. Mat. 8 (1970), $185-192$.

6. Bo Kjellberg, On the minimum modulus of entire functions of lower order less than one, Math. Scand. 12 (1963), 5-11.

Department of Mathematics, University of Otago, Dunedin, New Zealand 